\title{
Hidronefrosis (HN) neonatal en niños con hidronefrosis diagnosticada prenatalmente: ¿cirugía o tratamiento médico?
}

\author{
Gugliota A, Reis LO, Alpendre C, Ikari O, Ferreira U. \\ Servicio de Urología, Universidad Estatal de Campinas UNICAMP, Campinas, São Paulo, Brazil.
}

Actas Urol Esp. 2008;32(10):1031-1034

\section{RESUMEN}

HIDRONEFROSIS (HN) NEONATAL EN NIÑOS CON HIDRONEFROSIS DIAGNOSTICADA PRENATALMENTE: ¿CIRUGÍA O TRATAMIENTO MÉDICO?

Objetivos: No existen ni acuerdos ni criterios formales utilizados para establecer un tratamiento estándar en niños con diagnóstico de HN prenatal.

Métodos: Se analizó nuestra propia experiencia, además de los protocolos concernientes a este tema. Veintiocho (28) niños con HN unilateral fueron evaluados desde octubre de 1999 a octubre de 2005 y se midió el diámetro antero posterior de la pelvis renal (APDP).

Resultados: Los pacientes fueron sometidos a vigilancia con ecografía y renograma isotópico en 3, 6, 12 y 24 meses. Los resultados del tratamiento se valoraron siguiendo la clasificación de la Sociedad de Urología Fetal: HN ligera $(2 \mathrm{a} 5 \mathrm{~mm})$, moderada $(6$ a $10 \mathrm{~mm})$ y grave $(>10-15 \mathrm{~mm})$.

Conclusiones: Nuestras conclusiones son que la HN leve nunca debe ser intervenida quirúrgicamente, mientras que en la grave siempre debería realizarse cirugía. Por otra parte, la HN moderada, debe mantenerse bajo vigilancia con una ecografia y renograma isotópico durante dos años.

Palabras Clave: Prenatal. Hidronefrosis. Cirugía. Tratamiento médico. Fetal.

\begin{abstract}
POSTNATALLY HYDRONEPHROSIS (HN) IN CHILDREN WITH ANTENATALLY DIAGNOSED HYDRONEPHROSIS: SURGERY OR MEDICAL TREATMENT?

Objectives: There is no formal agreement or criteria utilized to establish a standard of treatment in children with antenatally diagnosed $\mathrm{HN}$.

Methods: We analyzed our own experience in addition to the protocols attending to this subject. Twenty eight (28) children with unilateral HN were assessed from October 1999 through October 2005 and the anteroposterior diameter of renal pelvis (APDP) was measured.

Results: Patients underwent surveillance with US and cintilography in 3, 6, 12 and 24 months. The treatment results were reviewed following the classification of Society of Fetal Urology: slight ( 2 to $5 \mathrm{~mm}$ ), moderate (6 to 10 $\mathrm{mm}$ ) and severe (>10-15 mm).

Conclusions: We concluded that slight HN would never be operated on while the severe ones would always be. The moderate HN, on the other hand, would remain under surveillance with US and cintilography for two years.

Keywords: Antenatally hydronephrosis. Surgery. Medical treatment. Fetal.
\end{abstract}

E 1 diagnóstico y tratamiento de la hidronefrosis (HN) en los recién nacidos y pre-adolescentes siguen siendo un tema polémico entre los urólogos, radiólogos, pediatras y nefrólogos ${ }^{1}$. En los últimos 20 años, se han diagnosticado numerosos casos de HN fetal gracias a la ecografía obstétrica (aproximadamente de 1,4 a 4,5\% de todos los exámenes) ${ }^{1,2}$.
Las causas más comunes de HN fetal son la obstrucción de la unión pielo-ureteral- pélvica (UPO), que constituye entre el 50 y el $60 \%$ de todos los casos y el reflujo vésico-ureteral, responsable del 20 al 30\% de esta anomalía. Existen otras posibles causas de hidronefrosis prenatal, pero son $\operatorname{raras}^{2,3}$.

No existen ni acuerdos formales ni criterios utilizados para establecer un estándar de tratamiento 
en niños con diagnóstico de HN prenatal. En la literatura se encuentran varios protocolos diferentes acerca de estos tratamientos, pero todavía no existe una práctica convencional que prevea la regresión completa del HN diagnosticado prenatalmente. Además, la falta de estudios prospectivos sobre la historia natural del HN prenatal, así como la total comprensión de los riesgos y beneficios de una intervención quirúrgica temprana en comparación con el tratamiento médico continuará estimulando la búsqueda de un manejo óptimo del $\mathrm{HN}$ post natal ${ }^{4-8}$.

Con el fin de abordar la cuestión previamente expuesta, nos decidimos a analizar nuestra propia experiencia, además de los protocolos que acompañan a este tema.

\section{OBJETIVO}

El objetivo es evaluar e interpretar los estudios retrospectivos e informar de nuestra propia experiencia con el diagnóstico y el tratamiento de $\mathrm{HN}$ postnatal. El propósito de este estudio es realizar la investigación y mostrar los parámetros empleados en el proceso de toma de decisiones de si el tratamiento óptimo para el HN es la terapia médica o la intervención quirúrgica.

\section{PACIENTES Y MÉTODOS}

Fueron evaluados un total de veintiocho (28) niños con $\mathrm{HN}$ unilaterales desde octubre de 1999 a octubre de 2005. Había 17 varones $(60,7 \%)$ y 11 niñas $(39,2 \%)$, examinados satisfactoriamente en este estudio. El rango de edad de los pacientes fue de 2 meses a 5 años, media 1,5.

Con el fin de detectar la causa o razón para la posible persistencia de $\mathrm{HN}$, todos los niños estudiados fueron diagnosticados con ecografía obstétrica, re-evaluados 7 días después del nacimiento y de nuevo a los 30 días.

Se midió el diámetro antero posterior de la pelvis renal (APDP), según la clasificación de la Sociedad de Urología Fetal: ligera (2 a $5 \mathrm{~mm}$ ), moderada (6 a $10 \mathrm{~mm}$ ) y grave (>10-15 mm).

Los niños con reflujo vesicoureteral, HN bilateral u otras anomalías urológicas (por ejemplo, válvula uretral posterior) fueron excluidos de este estudio.

Se realizaron renografías para diagnosticar la causa de HN, si era o no obstructiva, usando el renograma diurético con ácido dietilentriaminopen- taacetico o-DTPA Tc99. La función renal también se analizó mediante ácido dimercato-succinico oDMSA Tc99.

En todos los casos de HN obstructiva (obstrucción de la unión uretero pélvico) se complementaron con cistouretrografía miccional para excluir el reflujo vesico-ureteral.

La cirugía se realizó cuando se produjo un patrón de obstrucción en el renograma diurético con DTPA-Tc99, función renal $<40 \%$ con DMSA Tc99- y UTI repetitivo.

Todos los pacientes fueron sometidos a vigilancia con ecografía y renograma isotópico los 3, 6, 12 y 24 meses.

\section{RESULTADOS}

Diez de los veintiocho pacientes $(35,7 \%)$ experimentaron una recuperación completa de la $\mathrm{HN}$ a los siete días de vida, tal como se diagnosticó con el examen ecográfico. El mismo resultado se confirmó a los 30 días de vida con otro examen ecográfico. Seis pacientes $(21,4 \%)$ con HN moderada (>5 mm) fueron sometidos a vigilancia con ecografía y renograma isotópico. Cinco de estos seis no tenían patrón obstructivo en Tc99 DTPA-y la función renal $>40 \%$ en DMSA Tc99-, mientras que el restante tenía un patrón obstructivo y la función renal $>40 \%$.

Doce pacientes $(42,8 \%)$ fueron sometidos a cirugía (pieloplastia de Anderson-Hynes), logrando el abordaje posterior en ocho $(28,5 \%)$ y la realizando la clásica lumbotomía en los restantes 4 pacientes $(14,2 \%)$.

El seguimiento del grupo operado fue realizado utilizando ecografía y renograma isotópico a los 3,6 , 12 y 24 meses. Después de este período de tiempo, no hubo deterioro del cuadro clínico y no empeoró la función renal.

La APDP disminuyó en el 79\% de los operados. El 21\% de los pacientes post-cirugía sin cambios en la APDP, presentó aumento de la función renal del $31-34 \%$ al 40-48\% en el DMSA, con DTPA no obstructiva, mostrando la eficacia de la cirugía.

Por el contrario, en el grupo de tratamiento médico, tres (50\%) sufrieron el deterioro de la función renal acompañado con la ampliación de la $\mathrm{HN}$ en doce meses de seguimiento. Posteriormente, estos tres pacientes fueron sometidos a pieloplastia con buenos resultados (Fig. 1). 


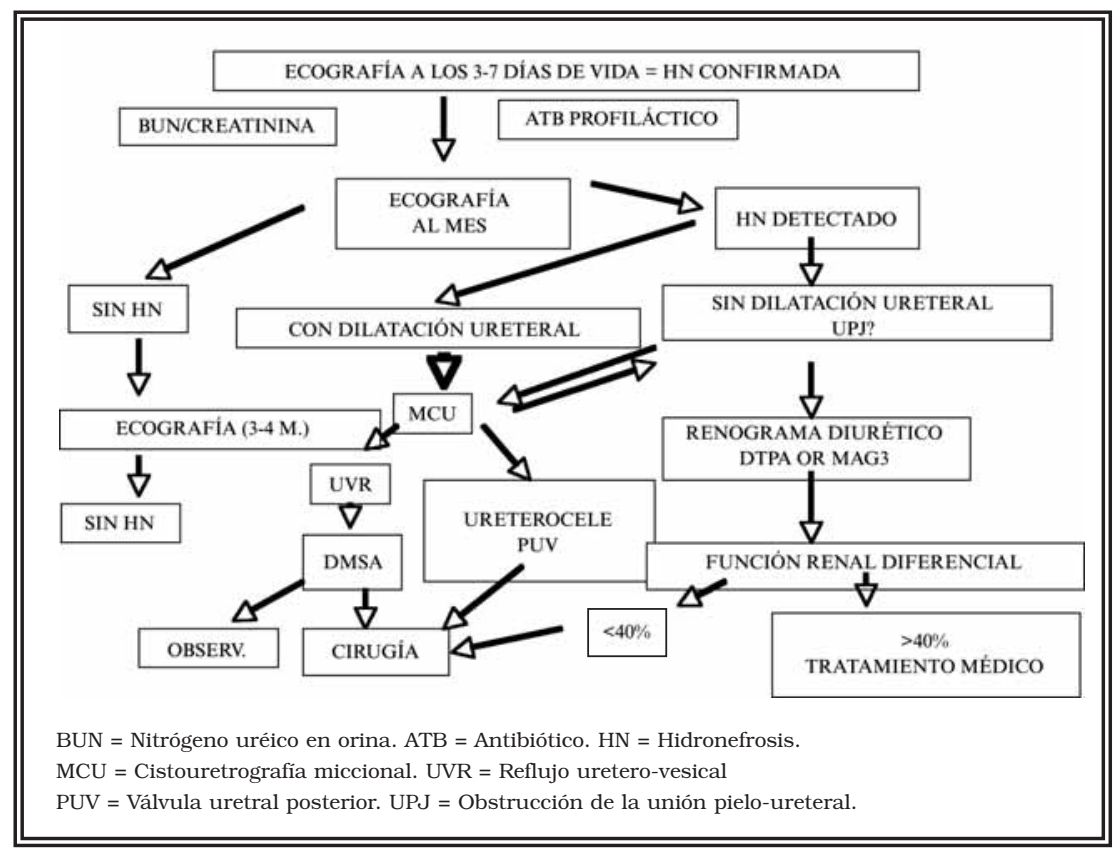

FIGURA 1. Algoritmo

\section{DISCUSIÓN}

La HN prenatal se presenta en el 0,25\% de todos los embarazos y es importante para la APDP estandarizar el diagnóstico de la dilatación ${ }^{2,3}$. La Sociedad Americana de Medicina Fetal ha formulado una escala útil para evaluar las dilataciones basada en los hallazgos ecográficos del útero: la APDP entre 3 y $5 \mathrm{~mm}$ se considera leve, 6 a $9 \mathrm{~mm}$ es moderada y mayor de $10 \mathrm{~mm}$ (en la $30^{\mathrm{a}}$ semana de gestación) o $15 \mathrm{~mm}$ (en la $33^{\mathrm{a}}$ ) se considera dilatación grave y, por lo tanto, se denominan dilataciones obstructivas ${ }^{9}$. Las dos últimas exigen una ecografía el renograma isotópico post-natal con el fin de determinar el tratamiento óptimo. Los estudios muestran recuperación espontánea en el 50\% de las dilataciones leves, el 15\% de las moderadas y ninguno en las graves ${ }^{9,10}$.

La elección óptima del tratamiento y su comienzo después del parto para los niños hidronefróticos diagnosticados prenatalmente sigue siendo un tema controvertido en la literatura médica. Las preguntas relacionadas con el tipo de riñones hidronefróticos que más se beneficiarían de una intervención quirúrgica y en cuales se deterioraría su función si se mantienen bajo vigilancia permanecen sin respues$\mathrm{ta}^{6,7}$. Por tanto, la evaluación de la función renal es un paso obligatorio para determinar qué riñones HN deberían ser intervenidos y cuales se mantendrían bajo vigilancia. Asimismo, la recuperación de la función renal en los riñones HN tras la cirugía o la intervención médica es el objetivo principal en el tratamiento de niños con obstrucción de la unión urétero-vesical ${ }^{8,10-12}$.

A pesar de que la literatura muestra buenos resultados estadísticamente, con ambos tratamientos médicos $\mathrm{y}$ quirúrgicos, una de las más difíciles situaciones, como señaló Dhillon, es distinguir qué niños han mantenido una buena función renal y cuales no ${ }^{12,13}$. Otra cuestión difícil es la duración que el tratamiento médico debe ser administrado. Todos los pacientes que reciben tratamiento médico deben ser valorados con ecografías y renogramas sistemáticos cada 3 o 6 meses durante 2 años. En el caso del aumento de la $\mathrm{HN}$ o el empeoramiento en la función renal $(<40 \%)$, se deberá realizar cirugía.

$\mathrm{El}$ seguimiento con ecografías y renogramas isotópicos durante dos años sigue siendo importante incluso después de la cirugía ${ }^{12,14-16 .}$

Contradiciendo las anteriores publicaciones, Chetin et al, informaron de que más del 50\% de los niños con diagnóstico prenatal de HN causada por la obstrucción de la unión utrero vesical que están bajo tratamiento médico necesitarán finalmente cirugía 17 .

En nuestro protocolo, existe una fuerte evidencia que a sugiere que los pacientes con HN contraída post natal, con una a función renal $<40 \%$ necesitarán tratamiento quirúrgico.

\section{CONCLUSIÓN}

El tratamiento ideal en HN congénitas está todavía en estudio. La historia natural de esta anomalía no está totalmente comprendida y no hay pruebas fiables para establecer criterios para elegir entre los tratamientos médicos o quirúrgicos.

Creemos que la HN ligera nunca debe ser intervenida, mientras que la más grave lo sería siempre, especialmente en los casos con ecografías repetitivas y diferencial de la función renal $<40 \%$. Por otra parte, la HN moderada, se mantendría bajo vigilancia con ecografías y renogramas isotópicos durante dos años. 


\section{REFERENCIAS}

1. Helin I, Person PH. Prenatal diagnosis of urinary tract abnormalities by ultrasound. Pediatrics. 1986;78(5):879-882.

2. Raynor BD. Routine ultrasound in pregnancy. Clin Obstet Gynecol. 2003;46(4):882-885.

3. Capello SA, Kogan Barry A. et al. Renal ultrasound has led to earlier detection and repair of ureteropelvic junction obstruction. J Urol. 2005; 174(4):1425-1428.

4. Ranjiv M. Antenatal hydronephrosis current evaluation and management. Indian J Urol. 2006;22:10-14.

5. Koff SA. Neonatal management of unilateral hydronephrosis. Role for delayed intervention. Urol Clin North Am. 1998 May;25 (2): 181-186.

6. Subramanian R, Kouriefs C, Dickson AP. Antenatally detected pelvi-ureteric junction obstruction: concerns about conservative management. BJU Int. 1999 Aug;84(3):335-338.

7. Ulman I, Jayanthi V, Koff SA. The long-term followup of newborns with severe unilateral hydronephrosis initially treated nonoperatively. J Urol. 2000;164 (3 Pt 2):1101-1105.

8. Chetin B, Rolle U, Puri P. Does delaying pieloplasty affect renal function in children with a prenatal diagnosis of pelvicureteric junction obstruction? BJU Int. 2002;90(1):72-75.

9. Fernbach SK, Maizels M, Conway JJ. Ultrasound grading of hydronephrosis introduction to the system used by the Society for Fetal Urology. Pediatr Radiol. 1993;23(6):478-480.

10. Harding LJ, Malone PS, Wellesley DG. Antenatal minimal hydronephrosis: is its follow up an unnecessary cause of concern ? Prenat Diagn. 1999;19(8):701-705.

11. Zaccara A, Marchetti P, La Sala et al. Are preoperative parameters of unilateral pielo-ureteric junction obstruction in children predictive of postoperative function improvement?. Scand J Urol Nephrol 2000;4:165-168.
12. Thomas Chi, Vickie A, Hiept N. Increased echogenicity as a predictor of poor renal function in children grade 3 or 4 hydronephrosis. J Urol. 2006;157:1898-1901.

13. Dhillon HK. Prenatally diagnosed hydronephrosis: The Great Ormond Street experience. BJU Int 1998;81Suppl 2:39-44.

14. DeGrazia E, Castagnetti M, Cimador M. Surgical or conservative treatment of congent hydronephrosis:Ten years' experience. Minerva Pediatric 2001;51(4):275-283.

15. Eskild JA, Gordon IF, Frokier J. Congenital unilateral hydronephros: A review of the impact of diuretic renography on clinical treatment. J Urol. 2005;173(5):1471-1476.

16. Coplen E, Austin PF, Yan YD, Jeffrey M. The magnitude of fetal renal pelvic dilatation can identify obstructive postnatal hydronephrosis and direct postnatal evaluation and management. $\mathrm{J}$ Urol. 2006;176(2): 724-727.

17. Chertin B, Pollak A, Rabinowitz R, Hain D, Farkas A. Conservative treatment of uretero-pelvic junction obstruction in children with antenatal diagnosis of hydronephrosis: Lessons learned after 16 years of follow-up. Eur Urol. 2006;49 (4): 734-738.

Correspondencia autor: Dr. L. Oliveira Reis Servicio de Urología

Universidad Estatal de Campinas UNICAMP

Campinas, São Paulo, Brazil.

E-mail autor: reisleo@unicamp.br

Información artículo: Original - Urología Pediátrica

Trabajo recibido: abril 2008

Trabajo aceptado: julio 2008 